\title{
The mother-daughter mating type switching asymmetry of budding yeast is not conferred by the segregation of parental $H O$ gene DNA strands
}

\author{
Amar J.S. Klar \\ Cold Spring Harbor Laboratory, Cold Spring Harbor New York 11724 USA
}

\begin{abstract}
The Saccharomyces cerevisiae HO gene, which encodes a site-specific endonuclease, is transcribed in the parent (mother) cell but not in the daughter (bud) cell. Consequently mother cells can switch their mating type whereas bud cells cannot. Whether or not the different capabilities of these cells are due to a nonrandom segregation of parental $\mathrm{HO}$ gene DNA strands to progeny cells is tested here by assaying the pattern of switching in cells where the $H O$ gene is inverted in the chromosome. In an inverted $H O$ strain, as is the case with the normal $\mathrm{HO}$ arrangement strains, only the mother cells were found to switch. Thus, the possibility of asymmetric segregation of parental $\mathrm{HO}$ gene DNA strands does not regulate the mother-daughter asymmetry of switching
\end{abstract}

[Key Words: Saccharomyces cerevisiae; HO gene; mating type switching]

Received May 22, 1987; revised version accepted September 23, 1987.

A fundamental question in developmental biology is how the progeny of a given cell division can acquire different developmental fates. The interconversion of the mating cell types of the budding yeast Saccharomyces cerevisiae has been used as an experimental system to address the question of developmental asymmetry between daughter cells. The alternate alleles of the mating type locus (MAT), called MATa and MAT $\alpha$, interchange by a transposition-substitution event whereby a copy of either the storage loci $H M L$ (a locus that usually contains unexpressed $\alpha$ information) or $H M R$ (a locus that usually contains unexpressed a information) is transmitted to the expressed MAT locus, resulting in a cell type change (for a recent review of the system, see Klar et al. 1984). The recombination event is initiated by a site-specific, double-stranded break found at the MAT locus (Strathern et al. 1982; Kostriken et al. 1983). The $H O$ gene, which is located on chromosome IV, encodes the site-specific "Y/Z endonuclease" which catalyzes the double-stranded break (Kostriken and Heffron 1984).

An interesting finding is that among a pair of sister cells only the older (parental) cell produces a pair of switched progeny in about $73 \%$ of cell divisions, whereas the newly born daughter (bud) cell never switches (Hicks and Herskowitz 1976; Strathern and Herskowitz 1979). This pattern of switching is due to the expression of the $H O$ gene in mother cells and its failure to be expressed in daughter cells (Nasmyth 1983). Why the $H O$ gene is expressed in mother cells and not in daughter cells is the subject of this study.

Regulation of the $\mathrm{HO}$ gene is complex. At least three separate regulatory systems act on the $H O$ gene such that it is only transcribed (1) in both a and $\alpha$ but not in a/ $\alpha$ diploid cells (Jensen et al. 1983); (2) only late in the $G_{1}$ phase of the cell cycle, and (3) only in mother cells and not in daughter cells (Nasmyth 1983). At least six trans-acting, positive regulatory genes called SWI (for switchingl, have been implicated in $\mathrm{HO}$ regulation. Recent studies indicate that some of the SWI products may activate $H O$ by antagonizing negative regulatory activities, of which there are at least two, in addition to the a/ $\alpha$ control (Jensen et al. 1983; Breeden and Nasmyth 1987; Nasmyth et al. 1987a,b; Sternberg et al. 1987; for review, see Klar 1987b). Over 1400 bp of DNA upstream of the transcription site are required for correct expression and regulation of the $H O$ gene (Nasmyth 1985).

One testable model that can account for restriction of HO gene expression to mother cells is that the chromatid inheriting the parental DNA strand containing a transcriptionally competent $H O$ gene is specifically segregated to the mother cell. This competence may be acquired by assembling a transcription complex on one of the $H O$ strands. In contrast, incompetent $H O$ is specifically segregated to the daughter cell (Nasmyth 1983; Brown 1984; Klar 1987a,b; Nasmyth et al. 1987b). Completion of the assembly process may require progression through the daughter's entire cell cycle, in which case the negative and positive controls discussed above could be required for precise regulation of the assembly process. This mechanism generates two nonequivalent sister chromatids. A precedent for such a model is provided by the recent studies with the distantly related fission yeast Schizosaccharomyces pombe. In this case it is argued that the potential for mating type switching is 
due to a strand-specific "imprinting" event of the recombinational substrate such that only one member of a pair of progeny cells can acquire the competence for switching. Consequently, it is suggested that two sister cells are developmentally nonequivalent since they inherit different strands of parental DNA (Klar 1987a).

Recent studies have demonstrated that a positive regulator of $H O$, called SWI5, is probably the key determinant for conferring mother-daughter asymmetry in budding yeast. Several observations are relevant. First, the SWI5 gene is expressed in a cell cycle-regulated fashion in both mother and daughter cells, but interestingly, this positive regulator is transcribed in the $S$ phase well after the window in which $H O$ is transcribed in late $\mathrm{G}_{1}$ (Nasmyth et al. 1987b). Therefore, the mother cell must asymmetrically inherit the SWIS gene product from the previous cell cycle to express $H O$. Second, Nasmyth et al. (1987b) have demonstrated that when the SWI5 gene is expressed throughout the cell cycle by placing it under the inducible GAL1 promoter, then both mother and daughter cells can switch efficiently. Third, the SWI5 protein binds in vitro to the HO promoter (D. Stillman and K. Nasmyth, per. comm.) and thereby blocks the action of the negative regulator(s) of $H O$ called SIN3 (Sternberg et al. 1987) and SDI1 (Nasmyth et al. 1987a). Fourth, stationary-phase mother cells, all of which are $G_{1}$ arrested, are capable of transcribing $H O$ when they undergo "START" following inoculation into fresh medium; that is, $G_{1}$ arrested mothers "remember" being mothers even after the block. Interestingly, a deletion of $H O$ upstream regulatory region between -788 and -1160 destroys its ability to be transcribed following $G_{1}$ arrest without affecting $\mathrm{HO}$ activity in subsequent cycles (Nasmyth et al. 1987b|. An explanation proposed by Nasmyth et al. (1987b) to account for these findings is that SWI5 may be inherited by mother cells only as a stable transcription complex whose stability is reduced in the case of the deleted promoter. Specifically, SWI5 may maintain a stable transcriptional complex at $\mathrm{HO}$ on only one chromatid following DNA replication. In this model the chromatid containing the competent $H O$ segregates to the mother cell pole at cell division.

A critical test of the strand segregation model is that the daughter cells should switch in those strains containing an inverted $\mathrm{HO}$ gene, whereas the mother cells should not. The results presented here rule out a strand segregation model involving $\mathrm{HO}$ for conferring developmental asymmetry between mother and daughter cells.

\section{Results}

Installing $\mathrm{HO}$ in either orientation in chromosome IV

The $H O$ gene was inserted next to the CDC36 locus located in linkage group IV by the following manipulations. The plasmid p519 contains the $H O$ gene on a 4.3$\mathrm{kb}$ Sau3A-EcoRI fragment cloned between the BamHI and EcoRI sites of pBR322 (see Fig. 1). This clone contains over $1700 \mathrm{bp}$ of sequences upstream from the tran- scription start site and contains the known regulatory sequences required for proper $\mathrm{HO}$ regulation (Nasmyth 1985). A 2.2-kb Sall-Xhol fragment containing the selectable yeast $L E U 2$ gene was cloned into the Sall site of p519 to generate the plasmid pAK32. A $2.7-\mathrm{kb}$ Sacl fragment, which contains a portion of the $C D C 36$ gene, was derived from the plasmid YRp7-CDC36.1 (Breter et al. 1983 ) and ligated into the unique SacI site of pAK32. This site is present at position - 1468 upstream from the HO transcription start (Nasmyth 1985). The resulting plasmid pAK37 contains the CDC36 SacI fragment in arbitrarily designated orientation I, while pAK36 contains the inverted orientation II. Both plasmids are $13.2 \mathrm{~kb}$ in size.

To place these constructs near $C D C 36$ in the chromosome, we used the observation that cleaved DNA molecules when introduced into yeast promote efficient homologous recombination at the cleaved ends (OrrWeaver et al. 1981). Therefore, to target the insertion next to $C D C 36$, plasmids pAK36 and pAK37 were cleaved at the unique Smal site present in the CDC36 SacI fragment (see Fig. 1). The linear DNA was used to transform strain DC150 (MATa leu2), and $\mathrm{Leu}^{+}$transformants were selected. Required transformants should integrate $H O, L E U 2$, and vector sequences, flanked by a single CDC36 SacI fragment on either side, into chromosome IV. In addition, since they contain an active $H O$ gene, these transformants should generate MATa/ MAT $\alpha$ diploid clones as a result of switching mating type and subsequent mating between cells of opposite type. The MATa/MAT $\alpha$ cells, unlike the parental strain, fail to mate and also can undergo meiosis and sporulation when transferred onto sporulation media. We found that most of the transformants indeed were stable $\mathrm{Leu}^{+}$ and had acquired the $H O$ gene since they had diploidized.

A Southern analysis of DNA isolated from several transformants demonstrated that the required genomic construction was obtained. Figure 2 demonstrates such an analysis of the parental strain DC150 and two transformants. The strain K734 (see Table 1 for complete genotype) was obtained from transformation with pAK37 DNA and strain K735 was derived from transformation with pAK36 DNA. The desired transformants can be identified by the pattern of hybridization to a genomic Southern to the YRP7-CDC36.1 (Breter et al. 1983) probe. When DNA is digested with BgIII, the parental 7.0-kb fragment, which contains the SacI fragment of the $C D C 36$ gene, should disappear, and instead two fragments of sizes $11.3 \mathrm{~kb}$ and $6.9 \mathrm{~kb}$ from orientation I (pAK37 transformation) and of sizes $14.0 \mathrm{~kb}$ and $4.2 \mathrm{~kb}$ from orientation II (pAK36 transformation) should be generated. $[7.0 \mathrm{~kb}+13.2 \mathrm{~kb}-2.0 \mathrm{~kb}=11.3 \mathrm{~kb}+6.9$ $\mathrm{kb}$ (orientation I) $=14.0 \mathrm{~kb}+4.2 \mathrm{~kb}$ (orientation II); the 2.0-kb BglII fragment contained within the $H O$ gene is not detected because the probe does not contain HO.] Predicted fragments were obtained (Fig. 2). In such a transformation only about one in three transformants showed a pattern consistent with the described construction. The rest of the transformants also resulted 

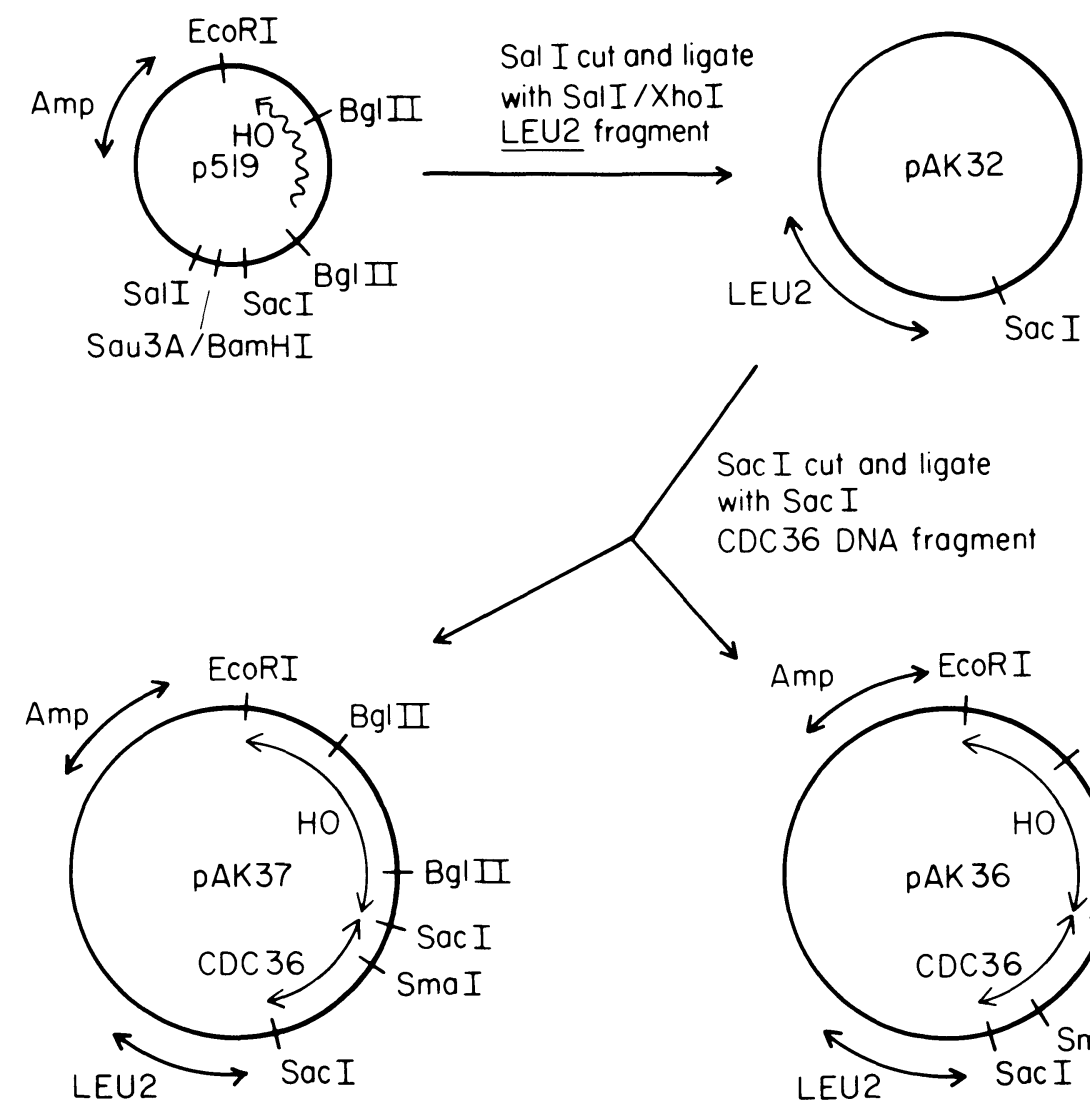

Sac I cut and ligate with Soc I CDC 36 DNA fragment

ORIENTATION I

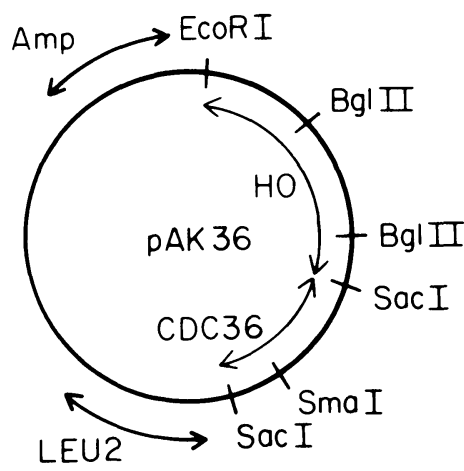

ORIENTATION II

\section{$\stackrel{\mathrm{IKb}}{\mathrm{Kb}}$}

Figure 1. The construction used for integrating $H O$ near the $C D C 36$ gene (see details in the text). The wavy line in plasmid p519 indicates the extent of the $H O$ gene transcript; the indicated restriction sites in $H O$ have been described earlier (J. Hicks, pers. comm.; Nasmyth 1985; Russell et al. 1986). Orientation refers to the orientation of the SacI fragment with respect to their sequences in plasmids pAK37 and pAK36. The figure is drawn approximately to scale.

from homologous recombination but contained multiple insertions (data not shown). Figure 3 diagrams the $H O$ inserts used for these experiments.

\section{The HO orientation does not affect the mother-} daughter switching asymmetry

The asci obtained from strains $\mathrm{K} 734$ and $\mathrm{K} 735$ were digested with glusulase and the resulting spores and their progeny were subjected to pedigree analysis (see Experimental procedures). Only $\alpha$ to a switches were tested. As presented in Table 2, mother cells of strain K734 switched in $35.2 \%$ of cell divisions while those of strain K735 switched with an efficiency of $27.3 \%$. Neither strain switched both their spores and daughter cells, and, therefore, followed the pattern observed with wild type HO-containing strains (Hicks and Herskowitz 1976; Strathern and Herskowitz 1979).

Both strains K734 and K735 that were used have the natural "inactive" allele of $H O$ (i.e., ho). To rule out the possibility that ho contributes some component essen- tial for switching, which may regulate the motherdaughter asymmetry, the pattern of switching of strains $\mathrm{K} 736$ an $\mathrm{K} 737$ was determined. Both the latter strains contain the hos : : TRPI allele in which most of the 5' coding region and part of the $H O$ promoter region are deleted (Nasmyth 1985). Again, results presented in Table 2 demonstrate that only mother cells of these strains switched regardless of the $\mathrm{HO}$ orientation.

Furthermore, strains $\mathrm{K} 738$ and $\mathrm{K} 739$, both of which contain $\mathrm{HO}$ at both $C D C 36$ and its indigenous location, also exhibit the wild-type pattern of switching (Table 2).

Taken together, these results demonstrate that the pattern of mating type switching, and thus the pattern of $H O$ expression, remains unaffected by (1) the $\mathrm{HO}$ orientation in chromosome IV, (2) the particular location of $H O$ in the chromosome, as well as (3) the presence of more than one copy of the $H O$ gene in the cell.

\section{Discussion}

This paper addresses the question of the means by which 


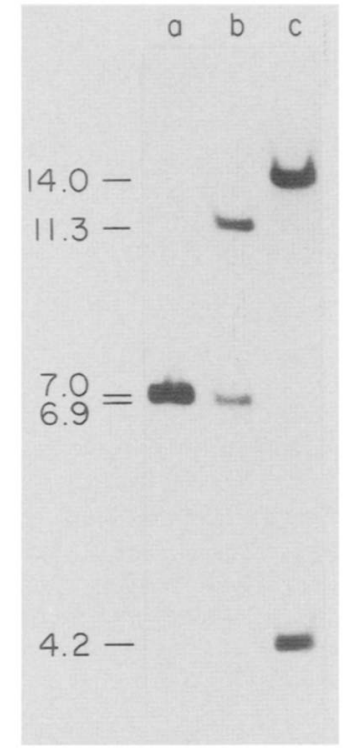

Figure 2. Southern blot analysis of BglII-digested DNA from parental strain DC150 and two transformants (K734 and K735) that contain the $H O$ gene inserted near the $C D C 36$ locus (lanes $a, b$, and $c$, respectively). The probe consisted of the radio-labeled plasmid YRP7-CDC36.1 DNA (Breter et al. 1983). The small difference in the sizes of $7.0-\mathrm{kb}$ fragment in lane $a$ and that of $6.9 \mathrm{~kb}$ in lane $b$ is not apparent in this figure, but the difference is clear when the DNA is separated by electrophoresis for a longer time (data not shown). The fragment corresponding to the TRP1 sequence present in the YRP7-CDC36.1 probe was run off the gel because of its small size. The numbers indicate DNA fragment size in kilobase pairs.

Table 1. Orientations of installed $\mathrm{HO}$ and genotypes of strains studied

\begin{tabular}{|c|c|c|}
\hline Strain & $\begin{array}{l}\text { Orientation of } \\
\text { installed } H O\end{array}$ & Genotype \\
\hline DC150 & - & 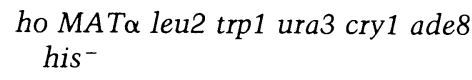 \\
\hline K734 & I & $\begin{array}{l}\text { ho/ho MATa/MATa leu2/leu2 trp1/ } \\
\text { trp1 ura3/ura3 cry1/cry1 ade8/ } \\
\text { ade8 } \text { his }^{-} / \text {his }^{-}\end{array}$ \\
\hline K735 & II & $\begin{array}{l}\text { ho/ho MATa/MATa leu2/leu2 trp1/ } \\
\text { trp1 ura3/ura3 cry1/cry1 ade8/ } \\
\text { ade8 } \text { his-/his }^{-}\end{array}$ \\
\hline K736 & I & 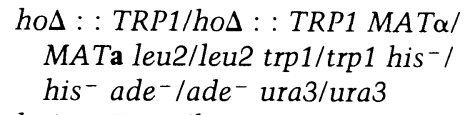 \\
\hline K737 & II & 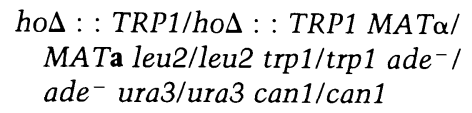 \\
\hline K738 & I & $\begin{array}{l}\text { HO/HO MAT } \alpha / M A T \text { a } \operatorname{trp} 1 / \text { trp1 } \\
\text { leu2/leu2(?) his-/his- ade-/ade } \\
\text { ura-/ura- cry1/cry1 }\end{array}$ \\
\hline K739 & II & $\begin{array}{l}\text { HO/HO MATa/MATa } \operatorname{trp} 1 / \operatorname{trp} 1 \\
\text { leu2/leu2(?) his-/his- ade-/ade- } \\
\text { cry1/cry1 }\end{array}$ \\
\hline
\end{tabular}

developmental asymmetry is generated between mother and daughter cells of yeast. The lineages of yeast cells are analogous to stem cell lineages; each cell division produces one cell incapable of switching and a second cell that has the competence to switch. Specifically, I tested whether this asymmetry is due to a strand-specific "imprinting" event at the $H O$ gene (which confers to it the competence to express) and whether such a marked strand is always segregated to the mother cell. Apparently the SWIS function is a key element in mother-daughter asymmetry and one suggestion for the mother cell specificity of $H O$ transcription is the asymmetric inheritance of the SWI5 product (Nasmyth et al. 1987b; Sternberg et al. 1987). Here it was tested genetically whether the SWI5 gene product, or some other determinant, is distributed nonequivalently to progeny cells forming a stable transcription complex to the $H O$ promoter by constructing strains in which the $H O$ gene, along with its upstream regulatory sequences which are known to bind in vitro to the SWI5 product, are inverted in the chromosome. In such a strain the motherdaughter switching asymmetry remained unaltered. Thus, it is concluded that the competence for $H O$ gene expression is not segregated with a particular strand of the $H O$ gene.

The observed efficiency of switching of mother cells in strains where $H O$ is placed next to $C D C 36$ gene is reduced significantly as compared with those containing $\mathrm{HO}$ at its orginal location. Although a model invoking a position effect control may be entertained to explain the reduced efficiency of switching, it is also possible that $H O$ and ho may compete for some regulator(s) whose concentration may be limiting, so that at low concentrations there is a reduced efficiency of switching. Consistent with this interpretation is the finding that those strains containing between two to three inserts of $\mathrm{HO}$ exhibit increased frequency of switching (nearly $2 \times$ ). Interestingly, a further increase in their number adversely affects the efficiency (data not shown). Alternatively, sequences $5^{\prime}$ to the SacI site may also be required for efficient transcription, because it is known that an insertion of a TRPI fragment into the SacI site at $H O$ reduces $H O$ transcription (K. Nasmyth, pers. comm.). Nevertheless, these results show for the first time that the SacIEcoRI fragment contains sequences that are sufficient for the whole repertoire of $H O$ control, though possibly not for full-level transcription.

One important point regarding the conclusion drawn needs to be addressed here. An alternate model may be considered in which the hypothesized strand segregation machinery may operate by binding directly to the $H O$ gene sequence. Specifically, such binding, for example, to the $H O$ sense strand, may align chromosome IV on the division plate so that the chromatid containing the competent $H O$ gene will always segregate to the mother cell. In this case inversion of the $H O$ gene is not expected to change the mother-dauther asymmetry. However, such a model is unlikely for the following reason. Some of the strains we used to test the model also contain the naturally occurring $\mathrm{HO}$ on the same chromosome. Should the hypothesized strand segregation mech- 


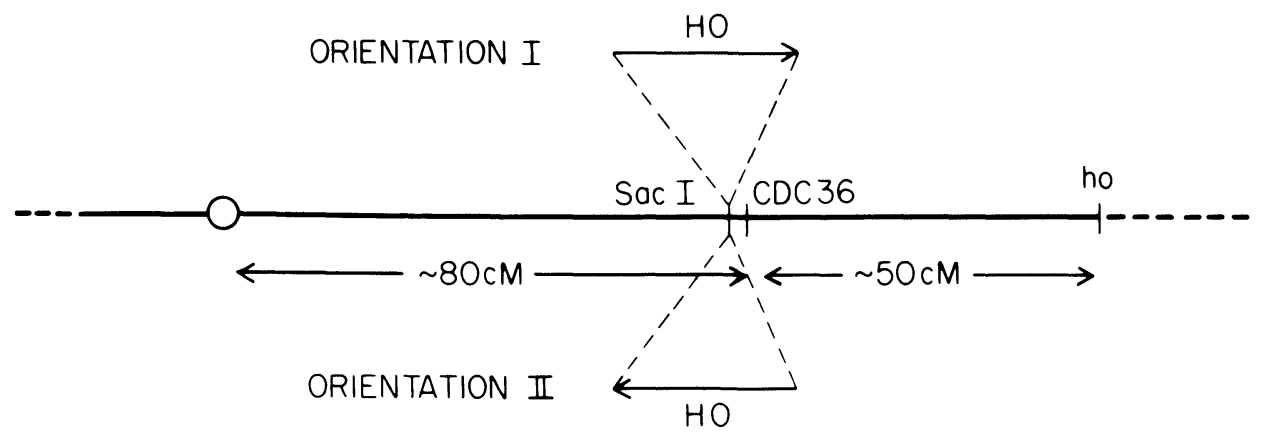

Figure 3. Diagrammatic representation of $H O$ inserted next to $C D C 36$ in chromosome IV. $H O$, along with the vector sequences plus the selectable marker LEU2, was inserted near CDC36 into the SacI site. Orientations of the inserted $H O$ with respect to centromere and the ho gene are not known and these are only arbitrarily designated. However, orientation I is opposite to that of orientation II. Thus, one of these inserts must be oriented opposite to that of the naturally occuring $H O$ gene. On the basis of meiotic recombination data, $C D C 36$ is located about $50 \mathrm{cM}$ away from the centromere (Mortimer and Hawthorne 1969). Mating type switching studies however, are conducted on mitotically growing cells that undergo very low levels of recombination. Consequently, for our purpose these elements are tightly linked. The centromere is represented by the circle.

anism operate by directly binding to the $H O$ sequences, then in a strain in which $H O$ is placed near $C D C 36$ in an opposite orientation to that of naturally occuring $H O$, both chromosome IV sister chromatids should be delivered to the mother cell. This constraint should lead to death of daughter cells. No such lethality was noticed during the pedigree analysis. In such strains both $\mathrm{HO}$ genes express only in mother cells, as indicated by their increased efficiency of switching (Table 2) compared with strains that contain $H O$ only at $C D C 36$.

To explain the mother-daughter asymmetry, two other types of models have been proposed by others. The first involves asymmetric distibution of a regulatory protein (such as SWI5), the other involving some other difference between mother and daughter cells, such as the difference in length of the $G_{1}$ phase of the cell cycle (Nasmyth et al. 1987b; Sternberg et al. 1987). Future studies should help us to differentiate between these and other possibilities.

\section{Experimental procedures}

\section{Yeast strains}

All strains used are of $S$. cerevisae. Construction of K734 and $\mathrm{K} 735$ is described in the text. The hos : : :TRPI allele was described earlier by Nasmyth (1985). The HO orientation refers to the $H O$ inserted near the $C D C 36$ locus.

Table 2. The effect of $\mathrm{HO}$ orientation on the pattern of mating-type switching

\begin{tabular}{lccccr}
\hline & \multirow{2}{*}{$\begin{array}{l}\text { HO } \\
\text { Strain }\end{array}$} & \multicolumn{2}{l}{ Switches per cell division } & \\
\cline { 3 - 5 } & orientation & mothers & daughters & spores \\
\hline K734 & I & $37 / 105(35.2 \%)$ & $0 / 111(0 \%)$ & $0 / 31(0 \%)$ \\
K735 & II & $78 / 286(27.3 \%)$ & $0 / 286(0 \%)$ & $0 / 41(0 \%)$ \\
K736 & I & $113 / 202(56 \%)$ & $0 / 223(0 \%)$ & $0 / 46(0 \%)$ \\
K737 & II & $27 / 63(42.8 \%)$ & $0 / 72$ & $(0 \%)$ & $0 / 13(0 \%)$ \\
K738 & I & $91 / 105(86.7 \%)$ & $0 / 97$ & $(0 \%)$ & $0 / 34(0 \%)$ \\
K739 & II & $54 / 75(72 \%)$ & $0 / 70$ & $(0 \%)$ & $0 / 33(0 \%)$ \\
\hline
\end{tabular}

Genetic analyses

All media for growth and sporulation and techniques for micromanipulation and tetrad analysis have been described (Mortimer and Hawthorne 1969). Pedigree analysis was conducted by a procedure described by Hicks and Herskowitz (1976). This procedure consists of testing the ability of cells to grow in the presence of an $a$-factor: the $\alpha$ cells grow while the a cells stop their growth and acquire a characteristic "shmoo" shaped structure. Pedigrees of $\alpha$ spores were followed for several generations by separating mothers from daughters by micromanipulation and observing the mating type by response to $\alpha$-factor.

\section{Biochemical analysis}

DNA from yeast cells was isolated by the procedure of Cryer et al. (1975). Standard procedures for manipulating DNA in vitro were used (Maniatis et al. 1982). For Southern blotting, $1 \mu \mathrm{g}$ of DNA was digested with the restriction enzymes and then subjected to electrophoresis through a $0.8 \%$ agarose gel. Transfer of DNA from the gel to nitrocellulose paper was done essentially as described by Southern (1975). The probes were nick-translated in the presence of $\alpha$-32 P-labeled deoxyribonucleotides and then hybridized as described (Maniatis et al. 1982). The DNAmediated transformation was accomplished by the glusulasegenerated spheroplast technique (Beggs 1978; Hinnen et al. 1978).

\section{Acknowledgments}

I thank J. Hicks who isolated and provided the $H O$ clone, $\mathrm{S}$. Reed for the $C D C 36$ clone, K. Nasmyth for a strain containing the hoD : : TRPI allele, K. Nasmyth and D. Stillman for communicating results prior to publication, members of Delbrück laboratory for discussions and comments on the manuscript, G. Fink and K. Nasmyth for their suggestions for improving the manuscript, K. Flynn and L. Miglio for excellent technical assistance, Dave Green and Mike Ockler for preparing the figures, and Laurie Lowman for preparing the manuscript. This work was supported by a research grant from the National Science Foundation, (DcB-8611960) and the Cold Spring Harbor Laboratory Robertson Fund.

\section{Note added in proof}

When $H O$ contained in pAK37 construct was integrated at LEU2 in chromosome III, again only mother cells switched 
$(37 \%)$ confirming that the mother-daughter asymmetry is indeed determined by sequences contained within the SacIEcoRI $H O$ fragment.

\section{References}

Beggs, J.D. 1978. Transformation of yeast by a replicating hybrid plasmid. Nature 275: 104-109.

Breeden, L. and K. Nasmyth. 1987. Cell cycle control of the yeast $H O$ gene: cis- and trans-acting regulators. Cell 48: 389-397.

Breter, H., J. Ferguson, T.A. Peterson, and S. Reed. 1983. Isolation and transcriptional characterization of three genes which function at start, the controlling event of the Saccharomyces cerevisiae cell division cycle: $C D C 36, C D C 37$, and CDC39. Mol. Cell. Biol. 3: 881-891.

Brown, D.D. 1984. The role of stable complexes that repress and activate eukaryotic genes. Cell 37: 359-365.

Cryer, D.F., R. Eccleshell, and J. Marmur. 1975. Isolation of high molecular weight DNA from yeast. J. Meth. Cell. Biol. 12: $39-44$.

Hicks, J.B. and I. Herskowitz. 1976. Interconversion of yeast mating types. I. Direct observation of the action of the homothallism (HO) gene. Genetics 83: 245-258.

Hinnen, A., J.B. Hicks, and G.R. Fink. 1978. Transformation of yeast. Proc. Natl. Acad. Sci. 75: 1929-1933.

Jensen, R., G.F. Sprague, and I. Herskowitz. 1983. Regulation of yeast mating type interconversion: Feedback control of $H O$ gene expression by the mating type locus. Proc. Natl. Acad. Sci. 80: 3035-3039.

Klar, A.J.S. 1987a. Differentiated parental DNA strands confer developmental asymmetry on daughter cells in fission yeast. Nature 326: 466-470.

- 1987b. Defermination of the yeast cell lineage (A minireview). Cell 49: 433-435.

Klar, A.J., J.N. Strathern, and J.B. Hicks. 1984. Developmental pathways in yeast. In Microbial development (ed. R. Losick and L. Shapirol, pp. 151-195. Cold Spring Harbor Laboratory, Cold Spring Harbor, New York.

Kostriken, R. and F. Heffron. 1984. The product of the HO gene is a nuclease: Purification and characterization of the enzyme. Cold Spring Harbor Symp. Quant. Biol. 49:89-96.

Kostriken, R., J.N. Strathern, A.J.S. Klar, J.B. Hicks, and F. Heffron. 1983. A site-specific endonuclease essential for mating type switching in Saccharomyces cerevisiae. Cell 35: 167174.

Maniatis, T., E.F. Fritsch, and J. Sambrook. 1982. Molecular cloning: A laboratory manual. Cold Spring Harbor Laboratory, Cold Spring Harbor, New York.

Mortimer, R.K. and D.C. Hawthorne. 1969. Yeast genetics. In The yeast, I (ed. A.H. Rose and J.S. Harrison), pp. 385-460. Academic Press, New York.

Nasmyth, K.A. 1983. Molecular analysis of a cell lineage. $\mathrm{Na}$ ture 302: 670- 676 .

- 1985. At least 1400 base pairs of $5^{\prime}$ flanking DNA is required for the correct expression of the $\mathrm{HO}$ gene in yeast. Cell 42: 213-223.

Nasmyth, K.A., D.J. Stillman, and D. Kipling. 1987a. Both positive and negative regulators of $H O$ transcription are required for mother cell specific mating type switching in yeast. Cell 48: $579-587$.

Nasmyth, K.A., A. Seddon, and G. Ammerer. 1987b. Cell cycle regulation of SWI5 is required for mother cell specific $H O$ transcription in yeast. Cell 49: 549-558.

Orr-Weaver, T.L., J.W. Szostak, and R.J. Rothstein. 1981. Yeasts transformation: A model system for the study of recombination. Proc. Natl. Acad. Sci. 78: 6354-6358.

Russel, D.W., R. Jensen, M. Zoller, J. Burke, B. Errede, M. Smith, and I. Herskowitz. 1986. Structure of the yeast $H O$ gene and analysis of its upstream regulatory region. Mol. Cell. Biol. 6: 4281-4294.

Southern, E. 1975. Detection of specific sequences among DNA fragments separated by gel electrophoresis. I. Mol. Biol. 98: $503-517$.

Sternberg, P.W., M.J. Stern, I. Clark, and I. Herskowitz. 1987. Activation of the yeast $H O$ gene by release from multiple negative controls. Cell 48: $567-577$.

Strathern, J.N. and I. Herskowitz. 1979. Asymmetry and directionality in production of new cell types during clonal growth: The switching pattern of homothallic yeast. Cell 17: $371-381$.

Strathern, J.N., A.J.S. Klar, J.B. Hicks, J.A. Abraham, J.M. Ivy, K.A. Nasmyth, and C. McGill. 1982. Homethallic switching of yeast mating type cassettes is initiated by a doublestranded cut in the MAT locus. Cell 31: 183-192. 


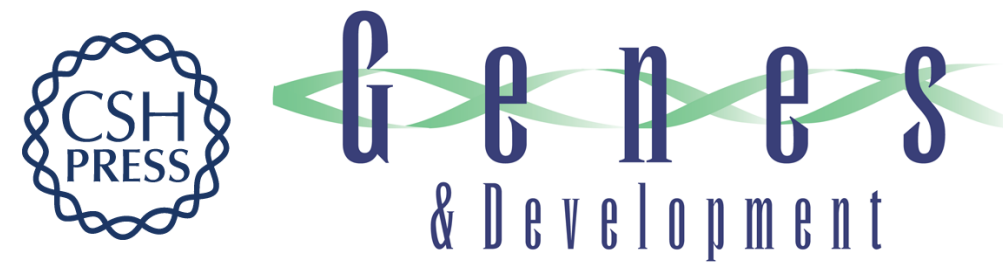

\section{The mother-daughter mating type switching asymmetry of budding yeast is not conferred by the segregation of parental HO gene DNA strands.}

\section{A J Klar}

Genes Dev. 1987, 1:

Access the most recent version at doi:10.1101/gad.1.10.1059

\section{References This article cites 22 articles, 7 of which can be accessed free at: http://genesdev.cshlp.org/content/1/10/1059.full.html\#ref-list-1 \\ License}
Email Alerting
Service
Receive free email alerts when new articles cite this article - sign up in the box at the top right corner of the article or click here.

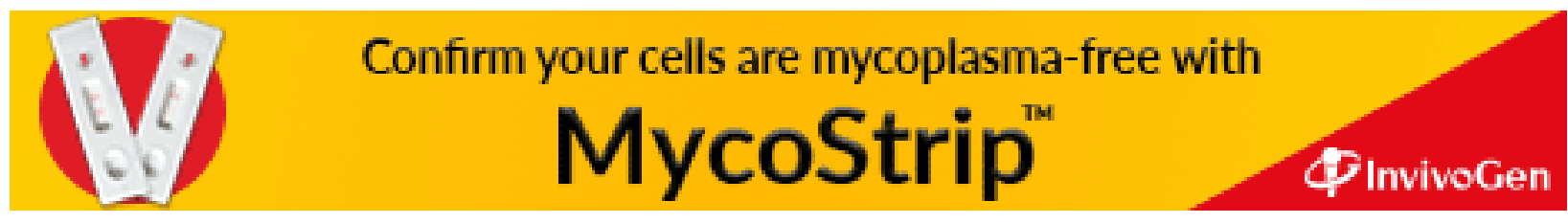

